\title{
James G. Arthur: AMS \\ 2017 Leroy P. Steele Prize for Lifetime Achievement
}

James Cogdell and Freydoon Shahidi, Guest Editors

Communicated by Steven J. Miller

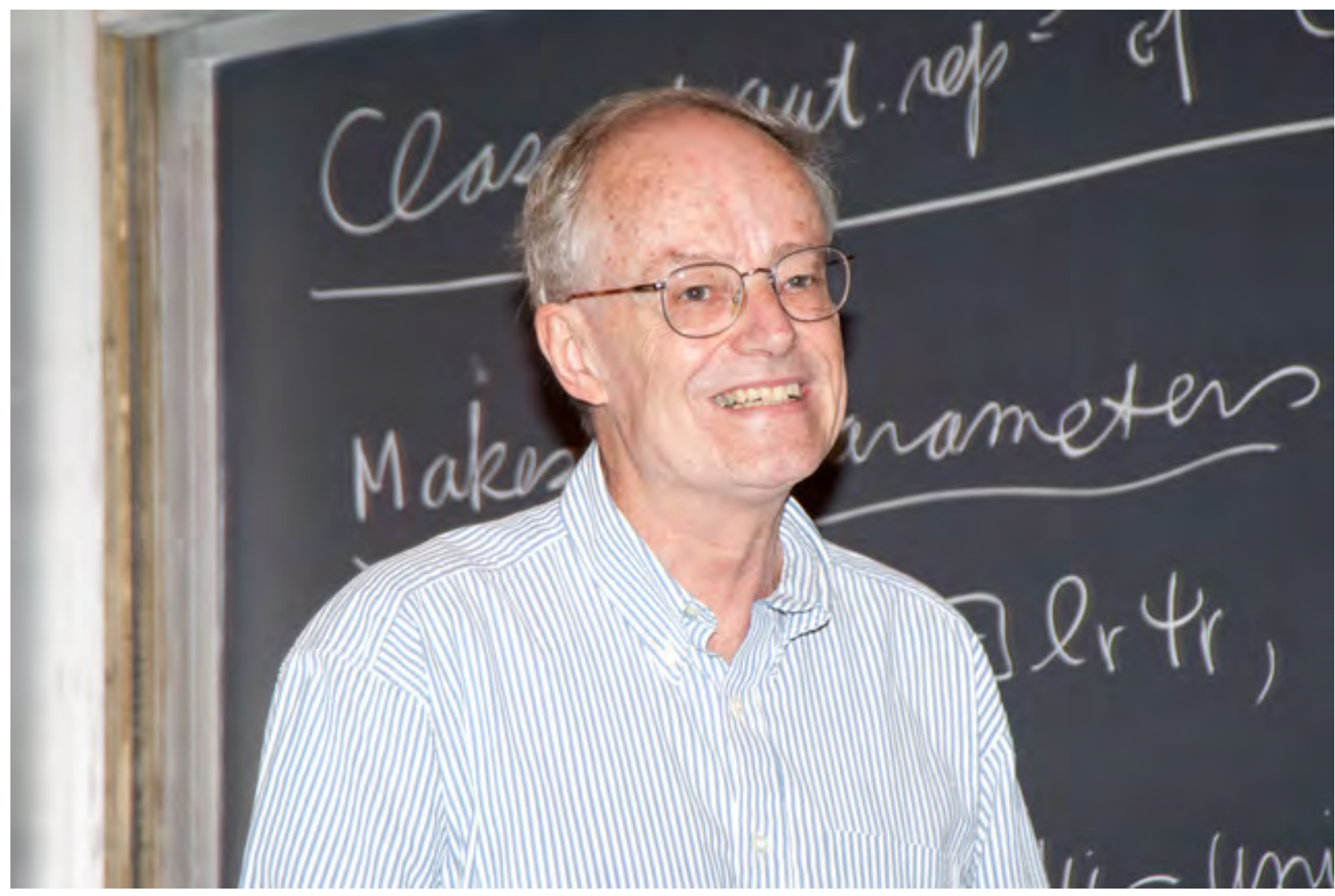

For permission to reprint this article, please contact:

reprint-permission@ams.org.

DOI: http://dx.doi.org/10.1090/noti1691 


\section{$\mathrm{J}$}

im Arthur's illustrious career, spread out over almost five decades, has contributed in a profound manner to our understanding of the theory of automorphic forms. He has single-handedly developed the trace formula, now known as the "ArthurSelberg trace formula." This trace formula is a powerful tool in understanding the spectrum of reductive groups over number fields and Langlands's Program. In particular, it can be used to establish important cases of Langlands's Functoriality Principle, one of the central questions in the theory of automorphic forms and number theory. Some of its most general cases are proved by Arthur himself.

Throughout his career he has received many honors, including the Canada Gold Medal for Science and Engineering (1999), the Wolf Prize in Mathematics (2015), and the Leroy P. Steele Prize of the AMS for Lifetime Achievement (2016). He was elected as a Fellow of the Royal Society of Canada (1980), a Fellow of the Royal Society of London (1992), a Foreign Honorary Member of the American Academy of Arts and Sciences (2003), and a Foreign Associate of the National Academy of Sciences (US) (2014).

James Greig Arthur was born in Toronto, Canada, in May of 1944. He studied at the University of Toronto, receiving his BSc and MSc in 1966 and 1967, respectively. After that he attended Yale University, where he received his PhD under Robert Langlands in 1970. Upon graduation he served as an instructor at Princeton (1970-1972), an assistant professor at Yale (1972-1976), and a professor at Duke (1977-1979) before joining the University of Toronto as a professor in 1979. He became a University Professor at Toronto in 1987, where he currently holds a Mossman Chair. Arthur's career has a significant service component, including terms as president of the AMS (2005-2006) and a member of the Board of Trustees of the IAS (1997-2007). He has also served on various panels and committees of the IMU, as well as of scientific societies in Canada and the US. On a personal level, he married Dorothy Pendleton (Penny) Helm on June 10, 1972, and they have two sons, James and David.

For this tribute we have asked several of his colleagues and associates to contribute their remembrances and assessment of this Lifetime Achievement of Jim Arthur.

David Vogan received his PhD in 1976 at MIT. He has devoted his career to studying harmonic analysis on reductive groups, much like Jim Arthur has. Here he provides a gentle introduction to some of the ideas that figure into the work of Jim Arthur.

James Cogdell is professor of mathematics at Ohio State University. His email address is cogde11@math. ohio-state. edu.

Freydoon Shahidi is Distinguished Professor of Mathematics at Purdue University. His email address is fshahidi@purdue. edu.

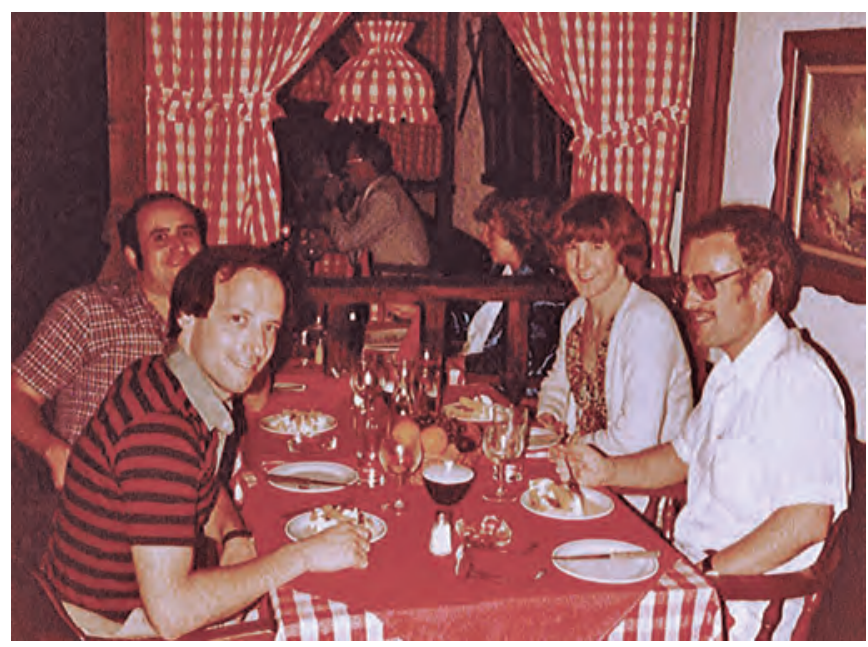

Freydoon Shahidi, Steve Gelbart, and Penny and Jim Arthur in Montreal, August 1980.

\section{David Vogan}

\section{Kernels of Beautiful Simplicity}

I first met Jim Arthur around 1975 when he was delivering a colloquium lecture at MIT. I'm sorry to say that I don't know exactly what the subject was: although I have dozens of pages of notes from talks by Jim over the years, I wasn't able to find notes for that first one. What he was working on at that time was what he's been working on his whole career: harmonic analysis on reductive groups. A reductive group is a group of matrices defined by polynomial equations and admitting no normal subgroups of unipotent matrices. What's allowed by this definition are things like $\mathrm{GL}(n, \mathbb{R})$ and $O(n)$ (defined in the first case by no polynomial equations and in the second by the $n^{2}$ quadratic equations $\left.{ }^{t} g g=I_{n}\right)$. What's ruled out are things like

$$
\left\{\left(\begin{array}{ll}
a & b \\
0 & d
\end{array}\right) \mid a \in \mathrm{GL}(p, \mathbb{R}), d \in \mathrm{GL}(q, \mathbb{R})\right\}
$$

(which has a normal subgroup

$$
\left\{\left(\begin{array}{cc}
I_{p} & b \\
0 & I_{q}
\end{array}\right)\right\}
$$

consisting of unipotent matrices).

Harmonic analysis is about understanding functions on $G$ or on homogeneous spaces for $G$ in terms of representations $\left(\pi, V_{\pi}\right)$ : vector spaces $V_{\pi}$ carrying actions of $G$ by linear transformations $\pi(g)$. Roughly speaking, harmonic analysis has two parts: a Fourier transform attaching to a function $f$ a linear algebra object $\pi(f)$ for each representation $V_{\pi}$ and Fourier inversion attaching a function to a collection of linear algebra objects $T_{\pi}$. The central example is the circle group $G=\left\{e^{i \theta}\right\}$. Irreducible representations $\pi_{n}$ of $G$ are indexed by integers. If $f$ is a

David Vogan is Norbert Wiener Professor of Mathematics at MIT. His email address is dav@math.mit. edu. 
function on the circle, the Fourier transform is $\pi_{n}(f)=a_{n}$, the $n^{\text {th }}$ Fourier coefficient of $f$ (a complex number). One of the basic theorems is that the Fourier transform of a smooth function on the circle is a rapidly decreasing function on the integers, that all such rapidly decreasing functions on the integers occur, and that the original function $f$ can be recovered by a convergent series

$$
f\left(e^{i \theta}\right)=\sum_{n} a_{n} e^{-i n \theta} .
$$

We all learn many (more subtle and difficult) variations on this theme, beginning with the Fourier transform description of square-integrable functions.

In the world of reductive groups, some of these ideas can (through the amazing work of Harish-Chandra and others) be carried through very nicely. There are families of representations parametrized by things like $\mathbb{Z}^{m} \times \mathbb{C}^{n}$, so it makes sense to speak of a Fourier transform that is "rapidly decreasing" or "holomorphic" in the representation parameter. Jim's earliest work concerns such natural extensions of classical results about Fourier transforms to reductive groups. (Of course making proofs of these results is much more difficult than formulating them!) He is most famous for his many results about the "trace formula," which is an extension to reductive groups of the Poisson summation formula.

I'd like to tell you instead about ideas that Jim developed beginning in the early 1980s concerning phenomena in reductive groups that have no analogue in classical Fourier analysis. I said that some representations of a reductive group come in families $\left(\pi(\nu), V_{\pi(v)}\right)$ with a parameter $v \in \mathbb{C}^{n}$. In the classical setting, all the spaces $V_{\pi(v)}$ are just $\mathbb{C}$, and so all the representations $\pi(v)$ are irreducible. For reductive groups, the spaces are infinitedimensional. The representations $\pi(v)$ are irreducible for most $v$, but for $v$ in certain affine hyperplanes in $\mathbb{C}^{n}$, $\pi(\nu)$ becomes reducible. This reducibility plays wonderful havoc with classical ideas. Functions that want to be holomorphic acquire poles along these hyperplanes, so interesting and complicated things happen when paths of integration are moved.

Langlands's description of harmonic analysis for square-integrable automorphic forms was phrased in terms of such behavior. He built the discrete part of the spectrum from the cusp forms (which are squareintegrable for natural geometric reasons) and the residual spectrum (which arises from poles of an appropriate highest order in the meromorphic continuation of the continuous spectrum). The difficulty was that there was no general understanding of when these highest order poles might occur.

That is the problem addressed by Jim's work beginning around 1982. Here is a version of the central idea. The Langlands Program describes representations of a reductive group $G$ in terms of semisimple elements in another complex reductive matrix group ${ }^{d} G$. The complex parameters for representations mentioned above are eigenvalues of these matrices. What Jim understood was that the appearance of poles should be controlled by the existence of nilpotent matrices commuting with

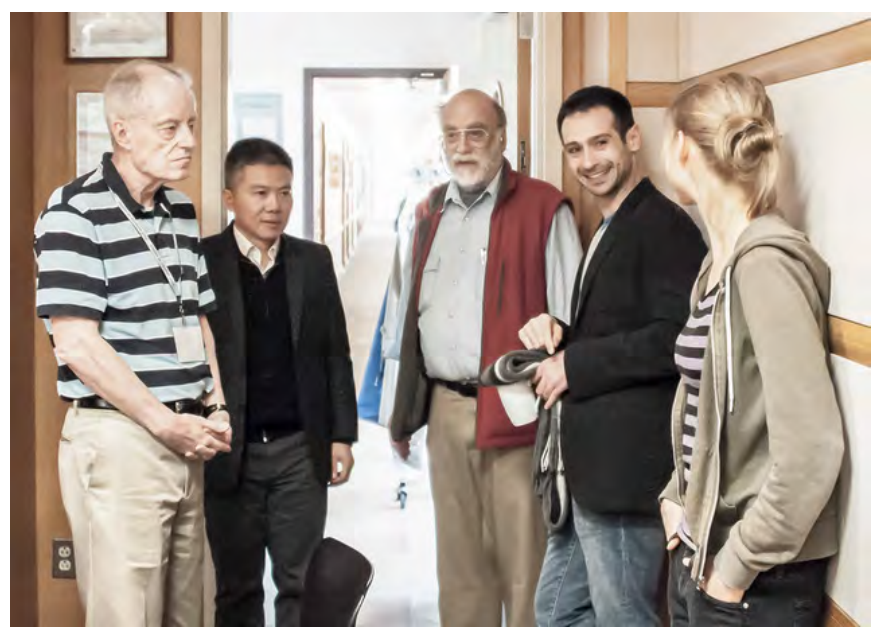

Jim Arthur, Ngô Bào Châu, Bill Casselman, Salim Ali Altuğ, and Jasmin Matz at the IAS conference in celebration of Langlands's eightieth birthday, October 2016.

Langlands's matrices. That is, poles should be related to elementary linear algebra questions about diagonalizing matrices with repeated eigenvalues.

Jim's work in the 1980s made this vague idea precise and drew powerful conclusions from it. He showed how to relate the idea to his work on the trace formula and to the Langlands-Shelstad ideas about stabilization and endoscopy. My friend Dan Barbasch and I have made our living since the 1980s on scraps from his table: Jim's ideas say where to look for interesting unitary representations of reductive groups over local fields, and so far he's been absolutely right.

The mathematics I love best addresses old and difficult problems using ideas with a kernel of beautiful simplicity. That's the mathematics that Jim does. He's an inspirational model, and I'm proud to count him as a friend.

To turn the Arthur-Selberg trace formula into a workable tool, one needs the "fundamental lemma." Langlands expected this to indeed be a lemma, but it has required much work and ingenuity to establish it. A proof of the fundamental lemma was provided by a young Vietnamese mathematician, Ngô Bào Châu, and it garnered him a Fields Medal in 2010.

\section{Ngô Bào Châu}

\section{The Laconic Professor Arthur}

It was some time in 2000 when I met Jim Arthur for the first time. I was then a young mathematician working in an area not directly related to his. At that time, I worked with Tom Haines, who was a postdoc in Toronto, on questions related to bad reduction of Shimura varieties.

Ngô Bào Châu is Francis and Rose Yuen Distinguished Service Professor of Mathematics at the University of Chicago. Hie email address is ngo@uchicago.edu. 
In a brief meeting I had with Jim, he handed me his recently completed papers on the stable trace formula. With his characteristic laconism, he said, "We need the fundamental lemma."

The fundamental lemma is a combinatorial identity discovered by Langlands in his study of points on Shimura varieties. In this context, the fundamental lemma is an ingredient in Langlands's strategy of expressing the Hasse-Weil zeta function of Shimura varieties in terms of automorphic $L$-functions. Slightly later, he realized similar combinatorial identities are needed in the stabilization of the trace formula for SL(2) that he worked out with Labesse. In the case of SL(2), the fundamental lemma can be proven by direct calculation of orbital integrals. In his lectures at École Normale Supérieure des Jeunes Filles, Langlands formulated the fundamental lemma in complete generality as a conjecture. Needless to say, nobody would care about these complicated combinatorial identities if they weren't important ingredients for establishing the stable trace formula. On the other hand, Jim's monumental work on the stable trace formula would remain conditional if the fundamental lemma wasn't proven. This little digression aims to provide some historical background to Jim's laconic sentence.

Jim's encouragement was important for me coming back to work full time on the fundamental lemma some years later. Some more years later, when I believed that I had the proof of that lemma, it was with a lot of emotion that I sent my preprint to Jim. His answer was again rather laconic: "You made my day." I still remember these words as a most treasured prize.

While working on the fundamental lemma, my understanding of Jim's work was rather superficial. My background is in algebraic geometry, which has very little to do with the arsenal Jim used in the construction of his trace formulas. Later, in learning Jim's work in more depth, I came closer to realizing all of its magnificence. There is little doubt that his construction of the trace formula for general reductive groups is one of the most important achievements of contemporary mathematics.

Arthur was a student of another highly influential Canadian mathematician, Robert P. Langlands. Langlands has had a great influence on the theory of automorphic forms and anointed the trace formula, as subsequently developed by Arthur, as the preferred tool for establishing the endoscopic cases of his Functoriality Principle. This is precisely what Arthur did for the classical groups. It has been said that Arthur sprang fully formed from Langlands's head, much as Athena did from Zeus's (but less dramatically), but in fact he only began work on the trace formula after his PhD. Here Langlands puts Arthur's work in the broad context of twentieth- and twenty-first-century mathematics.

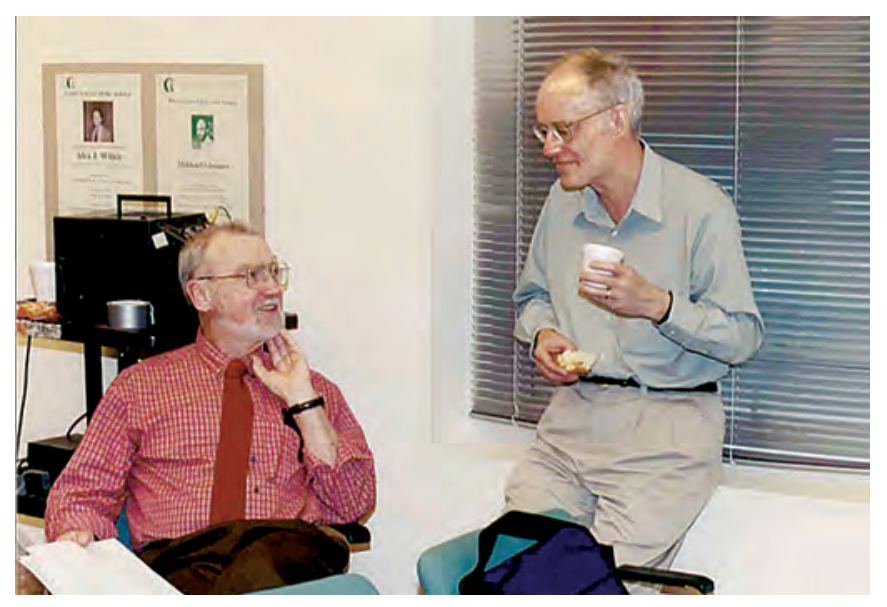

Robert Langlands and Jim Arthur at the conference in celebration of his sixtieth birthday, Fields Institute, October 2004.

\section{Robert P. Langlands}

\section{James Arthur}

The theory of automorphic forms as it evolved during the twentieth century contains one central theory, the theory over number fields, and two complementary theories of independent interest, the theory for function fields in one variable over finite fields and the geometric theory for function fields over complex algebraic curves. My views on the latter are explained in an article in preparation, “Об Аналитическом Виде Геометрической Теопии Автоморфных Форм” (An analytic view of the geometric theory of automorphic forms), and, so far as I can tell, for the former the decisive reference is the paper "Chtoucas pour les groupes réductifs et paramétrisation de Langlands globale" (Chtoucas for reductive groups and global Langlands parameterization) by Vincent Lafforgue. For analytic aspects of the theory over number fields, the principal contributor in recent decades has been Arthur.

He was preceded in the twentieth century by, above all, Hecke and Siegel, followed, with Hans Maass as intermediary, by Selberg. It is difficult to know just how much Selberg understood, but he was aware of the close relation between Eisenstein series and the spectral theory of differential equations on a half-line, a theory that appeared, for example, in a very early paper of Hermann Weyl on ordinary differential equations. A more striking, perhaps more imaginative, contribution of Selberg was the trace formula. This is a variant of the Frobenius reciprocity law for a subgroup $H$ of a finite group $G$, but in a context where the spectrum is in part continuous, so that serious analytic problems appear. For GL(2) they are not too difficult. A typical application appears in Chapter 16 of the text Automorphic Forms on GL(2) by JacquetLanglands. For groups of larger rank, the difficulties are

Robert P. Langlands is professor emeritus in the School of Mathematics of the Institute for Advanced Study. His email address is rpl@math. ias. edu. 
of a different degree. I began to think of the problem once I understood the theory of Eisenstein series, but with no success. Arthur began later, with no encouragement from me or, so far as I know, from anyone else, to study the problem. His thesis had been on quite a different matter. At the beginning of this century, thus almost thirty years after his initial efforts, I attempted to summarize, insofar as I had understood them, his contributions, which entail a great deal of technically and conceptually difficult analysis, so that they are, unfortunately, not easily accessible to those who most need to understand them: algebraicnumber theorists and arithmetically oriented algebraic geometers. The reference is "The trace formula and its applications," Can. Math. Bull. (2001). That summary was a major undertaking; a summary of his more recent contribution would be even more difficult.

He has himself described in a book, The Endoscopic Classification of Representations: Orthogonal and Symplectic Groups, which exactly these mathematicians can be encouraged to read, the developments of the intervening years, again for the most part, but not solely, contributions of Arthur himself. What are the major goals? They are a theory of functoriality and a theory of reciprocity. This is not the place to define precisely these theories or the nature of their relation one to the other. They can be considered, in particular, as expressions of the nonabelian class field theory, whose existence Artin had begun to doubt in 1946, when he concluded that "... whatever can be said about non-abelian class field theory follows from what we know now. ..." It is not a conviction, but a suspicion, not to be taken too seriously, that it was Chevalley and Artin who led mathematicians astray and that for these two theories-especially the first, about the second I have no clear idea-one has to return not to their expositions but to that of Helmut Hasse, Bericht über neuere Untersuchungen und Probleme aus der Theorie der algebraischen Zahlkörper, where the basic relation of class field theory appears as the equality of the number of solutions of two apparently different diophantine problems. I expect something similar to appear as a result of the trace formula and the related arithmetic in general. Arthur, in his book, does not reach this stage. He obtains, however, important and encouraging results for functoriality by an appeal to other arithmetic features of the problem, especially those related to the only partially developed and only partially arithmetic theory of endoscopy. Functoriality deals fully with the basic problem of analytic continuation of the Euler products of automorphic forms. It has, so far, nothing to offer to the Riemann hypothesis, although, on writing these lines, I recognize that I have never reflected on that question.

Moreover, in spite of the magnificent analytic achievements of Arthur, functoriality is far from a completely established concept. It can be considered as a part of harmonic analysis and as a part of analytic number theory. Its development in the second sense is only beginning. As a suggested introduction, I refer to the recent papers of Ali Altuğ, "Beyond endoscopy via the trace formula."

It is the issue of reciprocity that has the most attraction for number theorists. In brief, functoriality expresses the relation between automorphic forms on various groups according to principles that may be labelled galoisian, for the information is carried by groups and their relations. Knowing, as I wrote these lines, that unfortunately I had not found the time in recent years, even earlier, to study reciprocity and its several successes, among them Fermat's theorem, I spoke with my colleague Richard Taylor and, the conversation hardly begun, recognized that my grasp of the two theories, functoriality and reciprocity, and their relation was inadequate and misleading. Roughly speaking, it was explained to me that functoriality would aid in developing reciprocity. Namely, the first step would be to begin with a specific class of motives, those associated to the cohomology groups $H^{i}(X)$ of Shimura varieties, even to a specific class of Shimura varieties, and to show that they generated all the others. Functoriality established, the relevant operations, summands, sums, and products can be performed on both sides of the reciprocity-motives $\leftrightarrow$ automorphic forms-and thus lead to the desired reciprocity in general.

I explained my response to Taylor, who made it clear that although I had perhaps begun to understand the principles developed by him and his colleagues, I had exaggerated the possibilities offered by Shimura varieties and that there were many motives that were not accessible in this way, that like Thursday's child we had far to go. He did confirm, however, that "looking at the partial progress there has been on reciprocity in the last twenty-five years, functoriality, and in particular various instances of base change (and to a lesser extent endoscopy), have played a fundamental role. Without base change, and hence without Arthur's work, there would be many, many fewer theorems concerning reciprocity today."

Taylor had offered me reflections on a developing theory, but he did accompany them with references to specific work of him and Michael Harris, of Arthur-Clozel, and of Kottwitz. I did not have time to consult these before writing this essay, for which there was a short deadline, but it is clear that if $L$-functions are to play a role in the theory of diophantine equations, this will be in the context of functoriality, because the distribution of the Hecke-Frobenius parameters, thus the conjugacy classes in the group ${ }^{L} G$ that define the Euler products attached to automorphic forms, is determined not by a single $L$-function but by all those associated to a given automorphic eigenform.

Two of the mathematicians that probably have the greatest technical appreciation of Arthur's work and its influence are Colette Moeglin and Jean-Loup Waldspurger. Waldspurger did much early work on the fundamental lemma with an eye towards its eventual use by Arthur. Then together and separately, they have mined the consequences of Arthur's classification of local and global representations of the classical groups via the trace formula. Here they provide a more technical overview of the work of Arthur. 


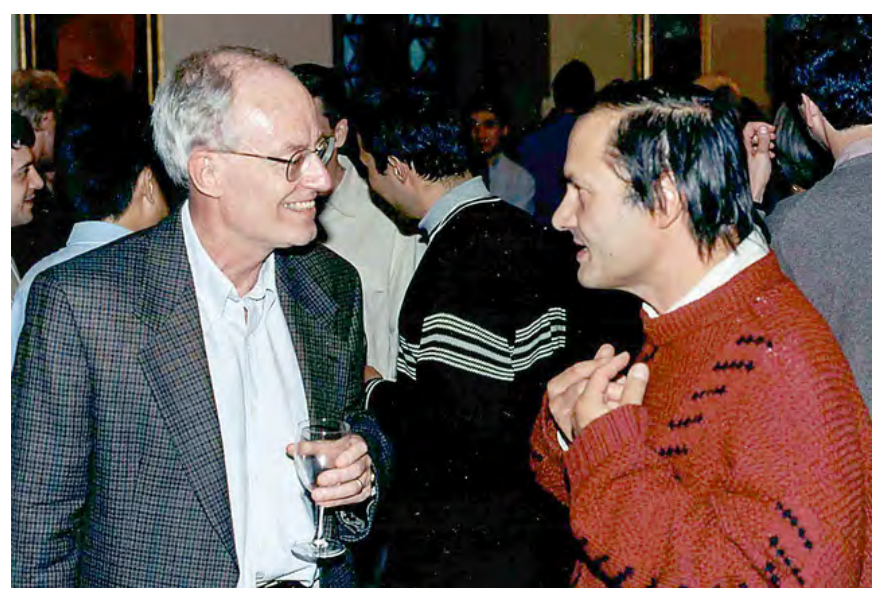

Jim Arthur and Jean-Loup Waldspurger at Arthur's sixtieth birthday conference, Fields Institute, October 2004.

\section{Colette Moeglin and Jean-Loup Waldspurger}

\section{The Work of J. Arthur}

Let $G$ be an algebraic group, connected and semisimple, defined over $\mathbb{Q}$, for example, $G=\mathrm{SL}(n), \mathrm{Sp}(2 n), \mathrm{SO}(n)$ for $n \geq 3$. Denote by $\mathbb{A}$ the ring of adèles of $\mathbb{Q}$. The group $G(\mathbb{A})$ acts by translation, on the right, in the vector space $L^{2}(G(\mathbb{Q}) \backslash G(\mathbb{A}))$. Denote by $L_{\text {disc }}^{2}$ the smallest closed subspace containing all the irreducible, closed, subrepresentations of $G(\mathbb{A})$. One of the goals of the theory of automorphic forms is to understand $L_{\text {disc }}^{2}$ as a $G(\mathbb{A})$ module. Of course, the theory also holds for groups like $\mathrm{GL}(n)$ or $\operatorname{GSp}(2 n)$; the only thing is to take care of the center in the definitions. The trace formula is one of the most powerful tools. This formula is an equality $J_{\text {geom }}^{G}(f)=J_{\text {spec }}^{G}(f)$. Here, $f \in C_{c}^{\infty}(G(\mathbb{A}))$ is any test function which takes value in the field of complex numbers. Both terms of this identity are complicated but contain a relatively simple principal term. For $J_{\text {spec }}^{G}$ it is $\sum_{\pi} m(\pi)$ trace $\pi(g)$, where $\pi$ describes all irreducible representations appearing in $L_{\text {disc }}^{2}$ and $m(\pi)$ is precisely the multiplicity of $\pi$ in $L_{\text {disc }}^{2}$. For the geometrical term, $J_{\text {geom }}^{G}(f)$, the principal term is $\sum_{\gamma} m(\gamma) I(\gamma, f)$, where $\gamma$ runs over the set of elliptic semisimple elements in $G(\mathbb{Q})$, up to conjugacy by $G(\mathbb{Q})$, and $m(\gamma)$ is a certain volume. This is one of the major works of Arthur to have been able to obtain this formula in full generality. The first formula obtained had the disadvantage of containing, in the complementary terms, some terms which were not invariant by conjugacy. Arthur has given a second

Colette Moglin is directeur de recherche at the CNRS, Institut de Mathématiques de Jussieu. Her email address is colette .moeg1in@imj-prg.fr.

Jean-Loup Waldspurger is directeur de recherche at the CNRS, Institut de Mathématiques de Jussieu. His email address is waldspur @math.jussieu.fr. formulation of the trace formula that contains only invariant distributions. But even this second formulation is not suitable for all the consequences we want, as it does not allow one to compare the formulas for different groups as wanted by the principle of functoriality of Langlands.

Let us look at the geometrical part of the trace formula. In the case where we can hope to establish a correspondence between the conjugacy classes in $G(\mathbb{Q})$ and the conjugacy classes in $H(\mathbb{Q})$, for two groups $G$ and $H$, this correspondence is in fact in general a correspondence for the conjugacy classes up to the action of $G(\overline{\mathbb{Q}})$ and $H(\overline{\mathbb{Q}})$ where $\overline{\mathbb{Q}}$ is an algebraic closure of $\mathbb{Q}$. This is called stable conjugacy. These two notions of conjugacy are different except for $\mathrm{GL}(n)$; an easy example can be found in $\operatorname{SL}(2, \mathbb{C})$. The idea of Langlands was that there should exist a stable version of the trace formula: $S I_{\text {geom }}^{G}(f)=S I_{\text {spec }}^{H}(f)$, at least if $G$ is quasi-split (which is a technical condition). This formula has to be similar to the previous invariant trace formula. On the geometric side, the principal term is a sum over the stable conjugacy class of elements in $G(\mathbb{Q})$. In the spectral side the representations have to be grouped in linear combinations which are stable, i.e., which annihilate the test functions whose stable orbital integrals are zero. This stable formula is what Langlands called the theory of endoscopy. The notion of endoscopic elliptic data has been defined (Langlands, Shelstad, Kottwitz, Labesse) and is denoted here by $\underline{H}$. It is to each such datum that we try to associate a stable trace formula (independently of $G$ of course) in such a way that we have an equality, for $*=$ geom or spec:

$$
I_{*}^{G}(f)=\sum_{\underline{H}} i(G, H) S I_{*}^{H}\left(f^{\underline{H}}\right),
$$

where $\underline{H}$ runs over the elliptic endoscopic data of $G$, $i(G, H)$ are explicit constants, and $f \mapsto f^{H}$ is the transfer coming from the correspondence between stable orbital integrals. Among the endoscopic data, we have the quasisplit form of $G$, and if $G$ is quasi-split, (*) is in fact the definition of $S I_{*}^{G}$, but one has to prove that the distribution so defined is stable. Arthur has established this theory in full generality. The proof of it not only occupies three papers of around one hundred pages but also a lot of preparatory papers. In fact, a lot of results had to be proved before in harmonic analysis and in the theory of representations. For example, the definition of weighted characters used the notion of normalized intertwining operators. But the theory, the actual state of knowledge, makes it impossible to compare the normalizing factors between a group and even one of its endoscopic data. Arthur has given a much better definition that we can control. But one of the most spectacular preparatory papers is the paper published in Selecta.

Arthur had previously made clear the importance of the elliptic representations (here the base field is a local one). These representations are the basis to obtain, via induction, all the tempered representations. Arthur proves that, for such representations, the stability and the endoscopic transfer can be read entirely on the subset of elliptic elements. A relatively direct consequence 
of such a result is the existence of the local spectral transfer. If $\underline{H}$ is an elliptic endoscopic datum of $G$, the Langlands-Shelstad transfer $f \mapsto f \underline{H}$ gives a function $f^{\underline{H}}$ for which only the stable orbital integrals are well defined. Dually, to a stable distribution for the endoscopic datum, $D^{H}$, there is associated a distribution $D$ for $G$ by the equality $D(f)=D^{H}(f \underline{H})$, and the local spectral transfer says that if $D^{H}$ is a finite stable linear combination of representations of $\underline{H}$, then $D$ is a finite linear combination of representations of $G$. The proof given by Arthur in Selecta is a local/global one and uses a simple form of the trace formula. This is a miniature of the general proof given in the three main papers, which we will now speak about. The difficulty of the stabilization is mainly on the geometric side. This comes from the way Arthur has made the trace formula invariant. The invariant form of the trace formula is no longer symmetric between a geometric and a spectral side; in fact both sides are now mixed. The weighted characters for tempered representation are now zero, but the information they have to carry has been put in the weighted invariant orbital integral.

Langlands and Kottwitz have handled the case of semisimple elliptic elements, and Arthur has to handle nonelliptic elements and especially those with a unipotent part. Already a semisimple nonelliptic element has complicated weighted orbital integrals, because this distribution contains information from the spectral side. Moreover, such an element is elliptic in a Levi subgroup, canonically attached to it, and the endoscopic data of this Levi subgroup also occur. The stabilization of the weighted orbital integrals attached to this kind of element occupies a great part of the proof. The proof is local/global using more than once the link between the geometric side and the spectral side. For the elements with a unipotent part, Arthur uses a descent method and a recurrence, and he is left with the purely unipotent elements. These elements are the only ones which can contradict the stability of the geometric side, but consideration of the spectral side proves that the way they will contradict the stability is not compatible with what is left as perhaps unstable on the spectral side. In fact, one can say that there are too few unipotent elements to block the process. Arthur has succeeded in walking around the unipotent part without computing it. This computation seems to be a very hard problem, and even though some progress has been made recently, this is perhaps the principal unsolved problem regarding the trace formula.

The stabilization of the trace formula has been a long-term job. It has been followed by another great deal of work, the explicit determination of $L_{\text {disc }}^{2}$ when $G$ is a classical group. This employs the stabilization of the trace formula and its twisted analogies. In this way, it is a whole part of the theory of automorphic forms which is due to Arthur.

As we noted earlier, Arthur's career had a significant service component, which included a stint as president of the AMS. We have asked a subsequent president, and one of Jim's longest associates, to comment on his term as AMS president.

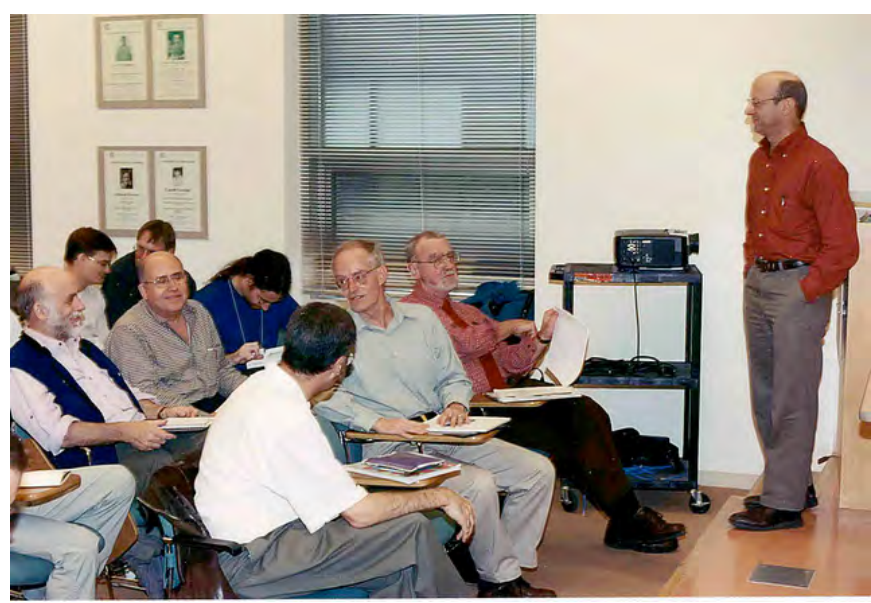

A lecture given at Arthur's sixtieth birthday conference at the Fields Institute, October 2004. On the stage is Steve Gelbart; in the first row are Bob Kottwitz, Jim Arthur, and Robert Langlands; in the next row are Bill Casselman, Freydoon Shahidi, and Lior Silberman; and in the back row, Tom Haines and Don Blasius.

\section{Eric Friedlander}

\section{Leading the AMS with a Light Touch}

The editors of this tribute to Jim Arthur have asked me to write something about Jim's role as president of the AMS. Tricky! Jim and I have been friends since we first arrived in Princeton in September 1970 as newly minted PhDs. In the almost half-century that has elapsed, we have had many amusing conversations and interesting mathematical discussions. The amusing conversations were often reflections upon our mathematical world and the delightful people who populate this world; those mathematical discussions typically involved my requests for enlightenment of Jim's very deep and difficult mathematics or Jim's requests for some targeted insight which I surely failed to satisfy. Looking back, I can't recall an unkind comment made by Jim (despite provocations), though there were some insightful comments about the idiosyncrasies of our mathematical community.

But what about Jim's AMS activities? First, Jim is a true gentleman and far more diplomatic than most mathematicians. Any critical remarks or complaints Jim would voice were shared in private, often with a big smile and a lowered voice. Perhaps he relied on me to some extent to rock the boat on difficult and thorny issues, such as the length of the Joint Prize Committee Ceremony or who would liven up AMS committees without causing mayhem. I presume that anyone with a strong opinion or a complaint who approached Jim during Jim's AMS presidency would have been fully satisfied with his

Eric Friedlander is the Dean's Professor of Mathematics at the University of Southern California. His email address is ericmf ausc.edu. 
response despite the fact that Jim held fast to his high standards and strong opinions.

My guess is that Jim might have suggested I write this "report" because of my role in assuring Jim that the "AMS green pages" given to members of the AMS Board of Trustees were not so impenetrable. Possibly, the color of these pages caused them to appear daunting; possibly, the size of sums considered was beyond our usual consideration (tens of millions of dollars); possibly, the detail and double bookkeeping of AMS finances at first threatened eyestrain and sleepless nights. But once Jim learned the tricks of asking a few innocent questions and appearing to be always on the correct page, he became a master.

AMS presidents are challenged by the need to appoint members to the uncountable number of AMS committees. Before this process began, Jim asked me to chair the AMS Committee on Committees. The dynamics of this committee proved somewhat challenging, but the entire process of selecting volunteers to advance the many positive efforts of the AMS was rewarding. Once again, humor leavened the burden, for we would discuss friends who were suitable as well as those who volunteered whom we did not know. We could proceed with some confidence, knowing that Bob Daverman's knowledge and wisdom would keep us from wandering too far astray.

Leading the Council is a delicate art, one which Jim mastered extremely well. Jim always kept the proceedings moving along with good humor, avoiding the pitfalls of lengthy personal anecdotes/remarks and curtailing discussions on topics not clearly relevant. Jim was invaluably guided by promptings from AMS Secretary Bob Daverman and the expertise of AMS Executive Director John Ewing. Flanked by Bob and John, exchanging handwritten notes and whispered comment, Jim had the AMS Council purring contentedly.

Indeed, I only recall one glitch. Once, after a meeting of the Executive Committee and Board of Trustees, we gathered to go out to enjoy a good dinner after all of our hard work. Having sorted out who had a car and who needed a ride, we arrived at the restaurant only belatedly to discover that we had left our leader (AMS President Jim Arthur) abandoned at the hotel. Of course, Jim rarely reminded us of the error of our ways.

Jim maintained a strong interest in preserving the important role that the AMS plays in advancing research mathematics through its publications, conferences, and prizes. A new distinguished lecture (the Einstein Lecture) is a consequence of Jim's efforts, and the evolution of AMS prizes and their selection committees owe much to Jim's interest.

So, what is my continuing impression of Jim Arthur and his AMS involvement? Reinforced by our continued "gossip sessions" and wry observations, my answer would be one of sense and sensibility, wit and good cheer.

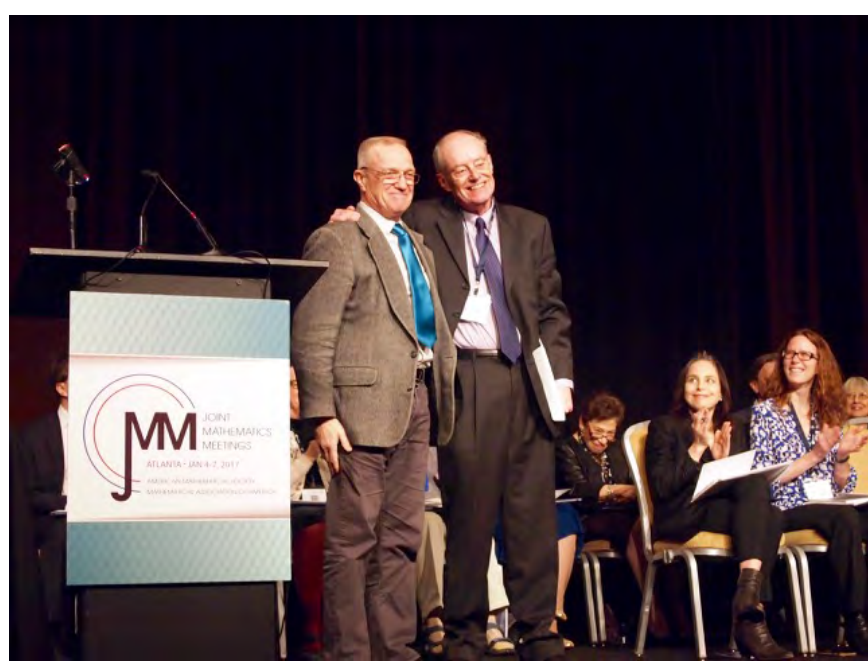

Jim Arthur receiving the Steele Prize for Lifetime Achievement from Robert Bryant, AMS Immediate Past President, at the Joint Mathematics Meetings in Atlanta, January 2017.

One thing we have not mentioned is Arthur's service as an advisor and mentor of graduate students. Arthur has been the advisor of eleven PhD students, one during his time at Yale (David DeGeorge, whom he shared with Bob Szczarba) and then ten while at the University of Toronto (Peter Mischenko, Jason Levy, Clifton Cunningham, Heng Sun, Cristina Ballantine, Paul Mezzo, Chao Li, Kam Fei Tam, Zhifeng Peng (whom he shared with Chung Pang Mok), and Bin Xu). One of his Toronto students was Cristina Ballantine, who received her PhD in 1998. Here she describes Arthur's talents as an advisor.

\section{Cristina Ballantine}

\section{In Defense of the Semicolon}

When mathematicians who are not number theorists ask me who my thesis advisor was, there might be a slight pause after I say Jim Arthur. Then, I just clarify: of the Arthur-Selberg trace formula. I don't need to add anything after that. Jim's name is by now somewhat interchangeable with the trace formula-the home of his mathematical life. I was a graduate student in Toronto for the better part of the 1990s. My first year there, he taught the graduate core course in real analysis. I loved analysis as an undergraduate and I felt comfortable with the subject. The way Jim taught it, however, had the effect of the right prescription glasses on a nearly blind person. I was in awe of his clarity.

At the end of the analysis course I knew I wanted to work with him. Soon, he became advisor to several students who had just started the PhD program. He was hoping to divide the work among us to establish the

Cristina Ballantine is professor of mathematics at the College of the Holy Cross. Her email address is cba11 ant@holycross. edu. 
trace formula for the metaplectic group. We knew so little about the trace formula that, as Jim's fiftieth birthday was approaching, we decided to make him a T-shirt with the trace formula on it. Needless to say, we gave up once we realized that it wouldn't fit on a T-shirt.

At the time, there was no introductory book on automorphic forms, and Jim decided that we would learn the basics through a reading seminar in which we studied some articles from the Corvallis proceedings. It wasn't an easy way to learn. It was frustrating, even discouraging at times. There was a lot we didn't understand, and this had never happened to us in our undergraduate days. Yet Jim kept telling us to not worry and that one day it would all click. He called this learning by osmosis. It's one of the skills I learned from him that I appreciate most. It requires patience and overcoming being uncomfortable with your own inability to understand. And it works; eventually, it clicks. While the seminar was painful for us students, talking about mathematics we didn't fully understand, Jim sat in the back and observed. What he was observing was not clear to me until about a year later when it was time to start working on an actual problem.

For personal reasons I had to leave Toronto for a year, and it made sense that I would work on a problem that was not directly related to the metaplectic group. From the student seminar, Jim could tell that I really enjoyed working with spherical functions and he found just the right problem for me. Ramanujan graphs had just entered the mathematical scene a few years before, and I started working on generalizing the construction to hypergraphs and analyzing the spectrum via automorphic forms. The key to my work was Rogawski's classification of the representations of the unitary group in three variables, yet I would have never been able to use it successfully without translating it first into the language of the Arthur conjectures, which, of course, I did following Jim's advice.

Initially, Jim's advising style was hands off. He told me I could come to him with questions whenever I had some. For me, this didn't work well. I am most productive when under pressure. Adrenaline is my friend. I am not sure what made him change his strategy, but when I came back to Toronto and he had just returned from a sabbatical in France, all his students met with him individually for a half hour on Fridays. If I remember correctly, my time slot was 12:30. These are some of the most memorable times of my life as a graduate student. Probably not all his students had the same experience, but for me these office visits were often a sort of metamorphosis. I would feel frustrated with my work and then, after talking to Jim, I was excited again to be thinking about this particular problem and confident that I could solve it. Altogether I was a pretty happy graduate student, in no small part due to Jim's guidance. I still have the notes he wrote during office hours. They are as clear as his lecture notes, though much shorter. It was also during the weekly meetings that I had a glimpse into the way his memory worked. He always knew precisely what article would be relevant to a particular question. There were piles of papers on the window sill in his office and he knew exactly where to find the one he was looking for. Once, I asked him a question about Hecke algebras, and after answering it he said, "Didn't you ask this two years ago?" He was also generous with wisdom about life in general. When I was expecting my first child, he told me how I shouldn't expect a white page I can write on, how babies have their own personalities and that's a wonderful thing. When I told Jim that I wasn't going to take any time off once the baby is born, he just said, "Wait until you hold that baby in your arms. We'll talk about it then."

The day I finished proving the main result for my thesis his reaction was, "Great. Now keep the momentum." Then came the writing of the thesis. Jim himself is a wonderful writer. I'm pretty sure that if he hadn't been a mathematician he would have been a novelist. His general advice was that the thesis should read like prose but that I should also make it short and snappy. The day after I gave him the first draft of my thesis, he called me at home to ask what I meant by the definition on page 5 . I explained, and ten minutes later he called back to ask another question about something on page 5. Another ten minutes later he called again and almost apologetically told me that I needed to rewrite the thesis. The next day I went into his office for more specific advice: no parentheses (if you need parentheses, it means you are not clear enough), do not start a sentence with the word And or a mathematical symbol, and no semicolons. I'm sure there was more, but I don't recall the details. Now, I can't help but repeat a story I told at Jim's sixtieth birthday conference. Years later, while searching for one of Jim's papers, among the many hits one read “'In Defense of the Semicolon' by James Arthur," a poem by his son of the same name.

I am extremely grateful that Jim Arthur took me as a PhD student. I learned a great deal of mathematics but also much about how to be part of the mathematical community, how to be gracious to my own students, and how to be happy and grateful for the chance to spend my life in mathematics.

\section{Photo Credits}

Montreal group photo courtesy of Freydoon Shahidi.

Photo of James Arthur receiving the Steele Prize courtesy of Asif Ali Zaman.

All other article photos by Jeff Mozzochi, courtesy of the Simons Foundation. 